\title{
Enhancing Masculine Features After Massive Weight Loss: Revisited
}

\author{
Dennis J. Hurwitz ${ }^{1}$ Ahmed A. Taha ${ }^{2}$
}

Published online: 5 August 2020

(C) Springer Science+Business Media, LLC, part of Springer Nature and International Society of Aesthetic Plastic Surgery 2020

Practical conceptual and technical innovations within "Enhancing Masculine Feature after massive weight loss" are the source of the article's popularity. This four-year follow-up updates those innovations, along with refinements.

The sheer magnitude of the skin deformity after massive weight loss demands a comprehensive approach far beyond isolated procedures for correction of gynecomastia, and skin laxity of the abdomen, flanks, buttocks, thighs, and arms. An organized plan including all those areas, as well as the boundaries between them, is necessary to obtain optimal results in as few stages as possible. In essence, a comprehensive approach first applied to craniofacial surgery is essential to body contouring surgery. For example, correction of the breast needs to include upper body lift often with the use of otherwise discarded nearby flaps, along with considerations of the long-term impact of circumferential lower body lift surgery.

As most patients presenting for total body lift surgery are women, the aesthetic issues related to men do not receive adequate attention. Furthermore, operations that work very well for women, such as a buried de-epithelialized inferior skin flap, should not be advocated for men for the correction of gynecomastia. These buried flaps leave inappropriate bulk between the nipple-areolar complex and inframammary fold (IMF). Furthermore, the

Dennis J. Hurwitz

drhurwitz@hurwitzcenter.com

1 Hurwitz Center for Plastic Surgery, University of Pittsburgh Medical Center (UPMC), 3109 Forbes Ave \#500, Pittsburgh, PA 15213, USA

2 Department of Plastic Surgery, Faculty of Medicine, Cairo University, Giza, Egypt anterior chest adherences should relate to the lateral and inferior borders of the pectoralis muscle and not to the breast. As such, the IMF should be obliterated, and not accentuated as occurs with a low transverse excision that leaves a scar along the IMF. Through ultrasound-assisted liposuction across the fold, followed by superior traction on the lower chest skin, IMF obliteration is achieved.

Extending the upper body lift transversely across the back, except for the most extreme cases, can be avoided with a lateral torsoplasty, which, because it dips inferior, is called a $\mathbf{J}$ torsoplasty. The $\mathbf{J}$ torsoplasty is an extension of the boomerang pattern excision of gynecomastia. Except for the most severely ptotic cases, both the gynecomastia and excess lateral bulk can be removed through these opposing oblique excisions encircling the nipple-areolar complex. These oblique excisions remove both transverse and vertical laxity, leaving a tight torso with the nippleareolar complex in the proper position. Traversing across the superior curve of the NAC visually breaks up the long meandering scar across the chest, making it less conspicuous. Care is taken not to extend the excision across the anterior midline and to avoid parasternal scar hypertrophy.

Despite the mechanical advantages of the Boomerang pattern with the J Torsoplasty, chest scars beyond the NAC perimeter are undesirable. Until recently, moderate skin laxity with breast ptosis in the massive weight loss patient requires excision. Skin tightening through bipolar radiofrequency and improvements in fat grafting are gamechangers. With the reintroduction of BodyTite ${ }^{\circ}$ (InMode, Yackom, Israel) to our practice in 2017, mild and moderate cases of gynecomastia were managed. There are some with impressive results and others not satisfied even after three effective attempts to shrink their breasts. They have been pleased with smaller skin resection. When BodyTite ${ }^{\circledR}$ 


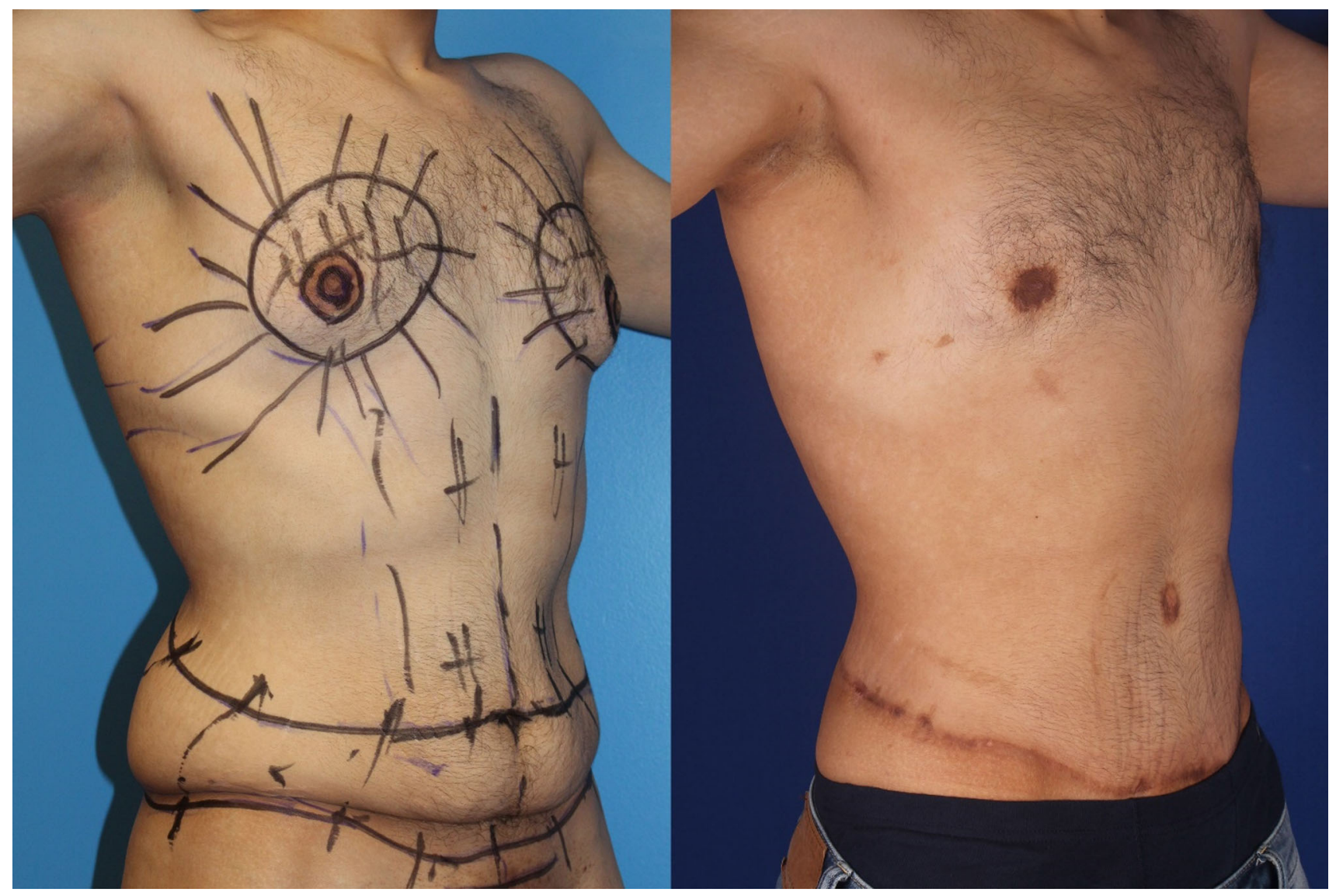

Fig. 1 Right anterior and left posterior obliques views of Combining Lipoabdominoplasty and oblique Flankplasty combined with Vaserlipo of gynecomastic with periareolar mastopexy followed by

treats the ptotic gynecomastia and chest in conjunction with an Oblique Flankplasty with lipoabdominoplasty (OFLA), considerable skin laxity and contour deformity of the entire torso can be corrected (Figs. 1, 2).

Bilateral Oblique Flankplasty was first applied to successfully avoid problems inherent with the male lower body lift (LBL). The standard LBL updated by Lockwood [1] has a complication rate approaching 50\% and not only fails to deflate and tightened the flanks but was bedeviled by deep lateral gluteal depressions, elongation of the intergluteal cleft, recurrent saddlebags and flattening of the buttocks [2]. Effective Flankplasty requires deep and wide resection of flank skin and fat to lumbodorsal fascia and
BodyTite ${ }^{\circledR}$ of both the breasts and entire chest. Left: preoperative markings. Right: 18 month result. Bipolar radiofrequency treatment was effective and avoided large hyperpigmented scars over the chest

External Oblique muscle to reverse lower back skin laxity and bulges and suspends the lateral buttocks and upper thighs. Recently appreciated is the capacity of OFLA to tighten all mid-torso skin, with the assistance of indirect undermining through liposuction. Upon resection of the dense and tight adherences of the flanks, skin closure tightens lax skin throughout mid-back, lateral chest and epigastrium. In all but the most severe cases of epigastric transverse skin laxity, the non-aesthetic, complication prone Fleur-de-Lis abdominoplasty can be avoided. Significant modification of the original mostly transverse flank excision pattern better tightens epigastric skin laxity. The paraspinous portion of the Flankplasty is drawn vertically, 


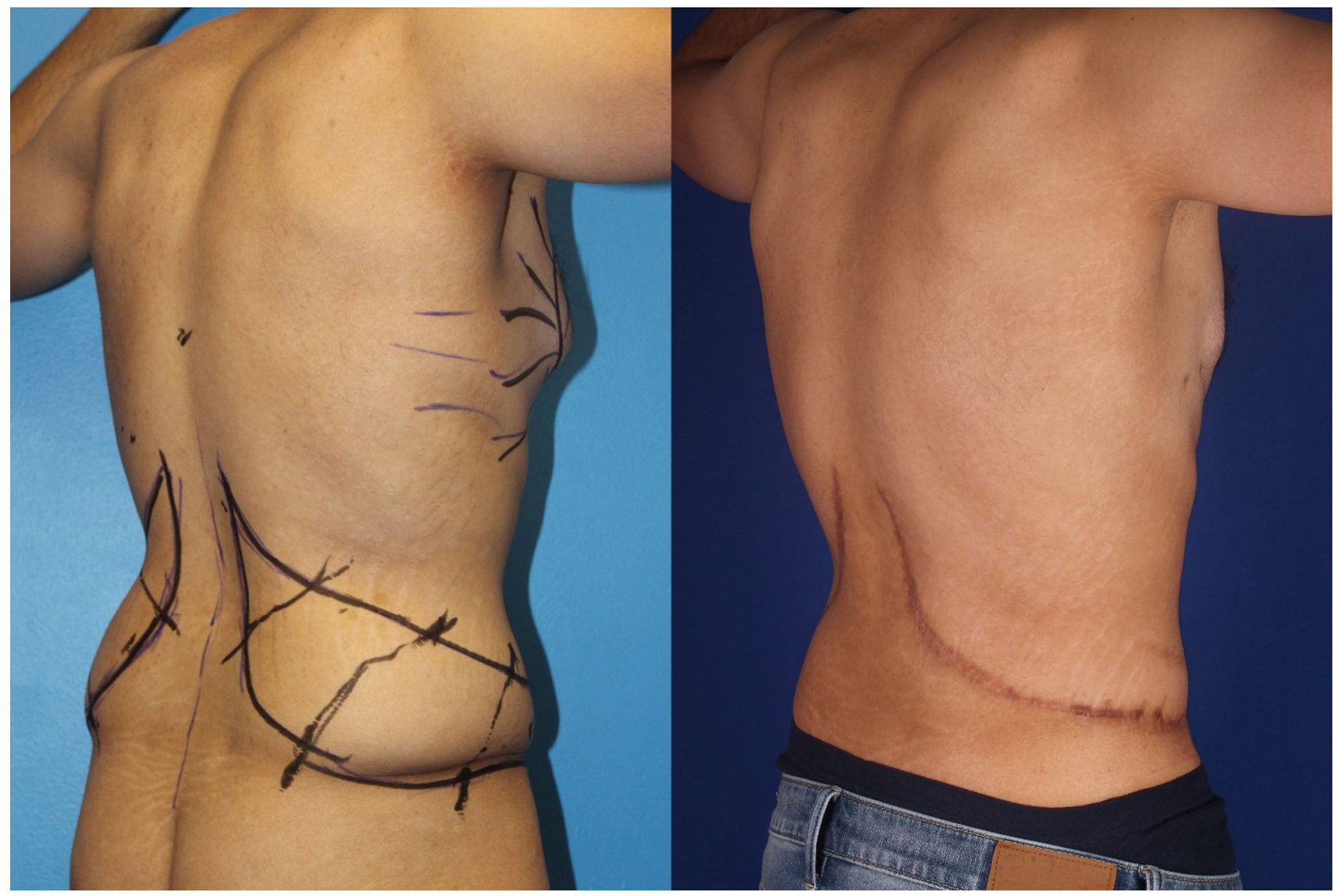

Fig. 2 Right posterior oblique views of Combining Lipoabdominoplasty and oblique Flankplasty combine with Vaserlipo of gynecomastic with periareolar mastopexy followed by BodyTite ${ }^{\circledR}$ of both

leaving a pattern resembling a hockey stick. Due to lax lateral and epigastric skin adherences, the surgeon pulls posteriorly on the abdomen prior to the abdominoplasty as if tightening the back of a vest. Assuming the patient has the typical male barrel-shaped chest and narrow pelvic rim, an aggressive soft tissue flank resection leaves a straight waist and discernable hips, dominated by skin-tight broad shoulders, back, and chest. In women with typical tapered lower ribs and broad pelvic rims, a deep and smoothly transitioned narrow waist is created over well-defined hips.

Since Hurwitz's article, liposculpture has become a valuable tool for masculinizing the upper torso. Based on the pioneering negative and positive space concepts of the breasts and entire chest. Left: preoperative markings. Right:18 month result, showing a properly shaped waist and tight torso

Hoyos and Millard [3], our fat injection in the pectoral and deltoid regions, together with focused liposuction, reliably creates the sought after sculptured masculine V-tapered look. As muscular men do have cleavage, its creation is essential (Fig. 2). The amount of fat needed for sculpturing the upper torso is around $1000 \mathrm{cc}$ of pure decanted fat. The injection is done using a Leur-lock $20 \mathrm{cc}$ syringes, fitted to $25 \mathrm{~cm}$ in length, $3 \mathrm{~mm}$ curved cannulas to accommodate muscle convexity. Over injection overcomes likely increased fat resorption due to mobile structures as the muscles [3]. The retention rates for the pectoralis are $60-70 \%$, while the deltoids appear about $80 \%$ [4]. 
Fig. 3 Creating the male cleavage through strong liposculpture of the midline together with fat grafting to the pectoral muscles
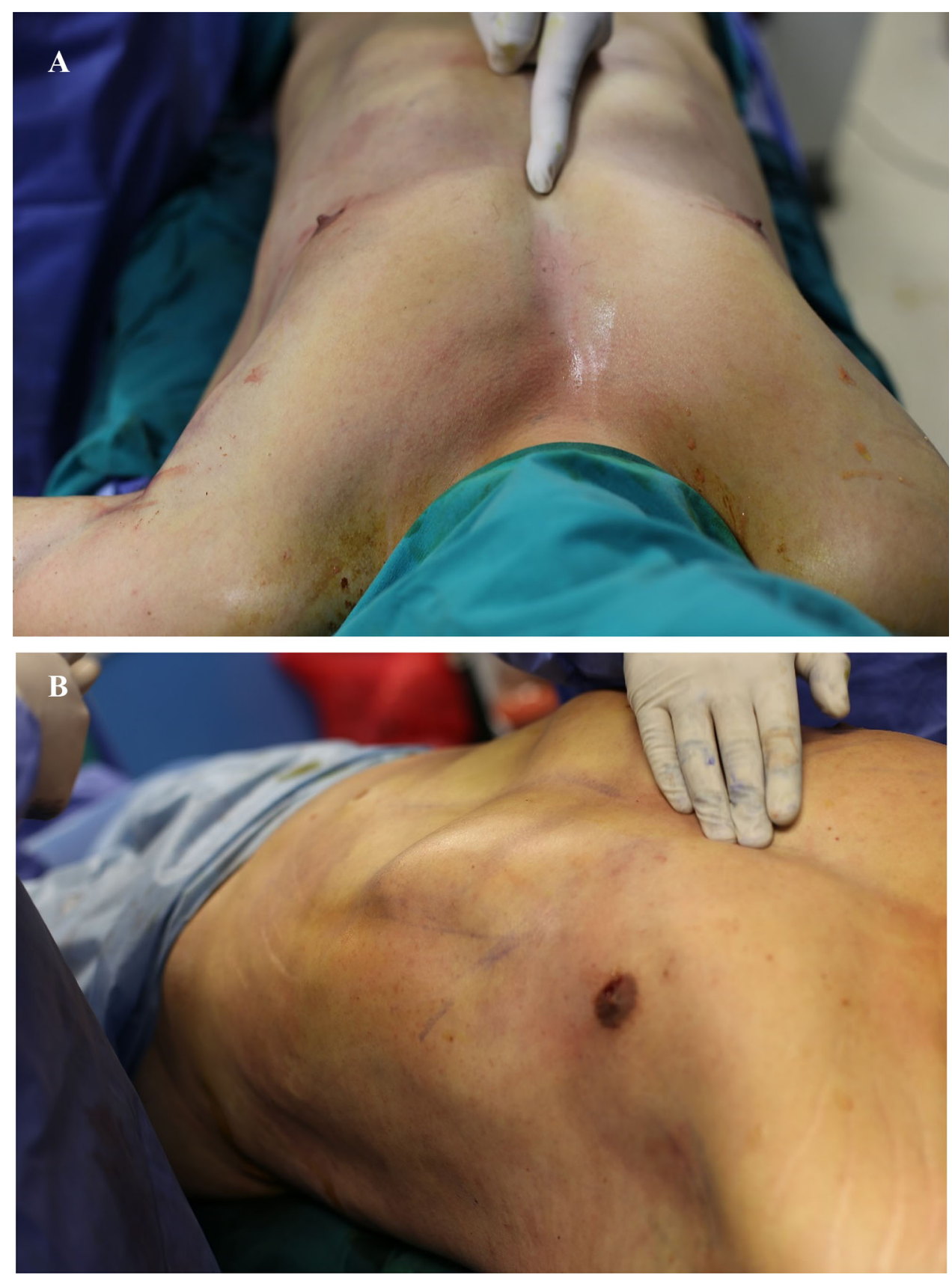

Liposculpture without skin excision is an option after mild weight loss (15-20 lbs). With VASER and powerassisted liposuction (PAL), healthy male skin usually recontours over fat expanded musculature [5-9] (Fig. 3). Additionally, males are not subject to the hormonal changes and skin stretch during pregnancy and childbearing $[10,11]$.
In selected cases, mildly obese patients can be the right candidate for liposculpture [12]. There BMI should be less than $35 \mathrm{~kg} / \mathrm{m}^{2}$, with no comorbidities, excellent skin quality, and motivated enough to maintain the longevity of their results post-surgery. We, as surgeons, place that kind of patient back on track. With the right motivation for their new bodies, they usually continue their ongoing journey to 

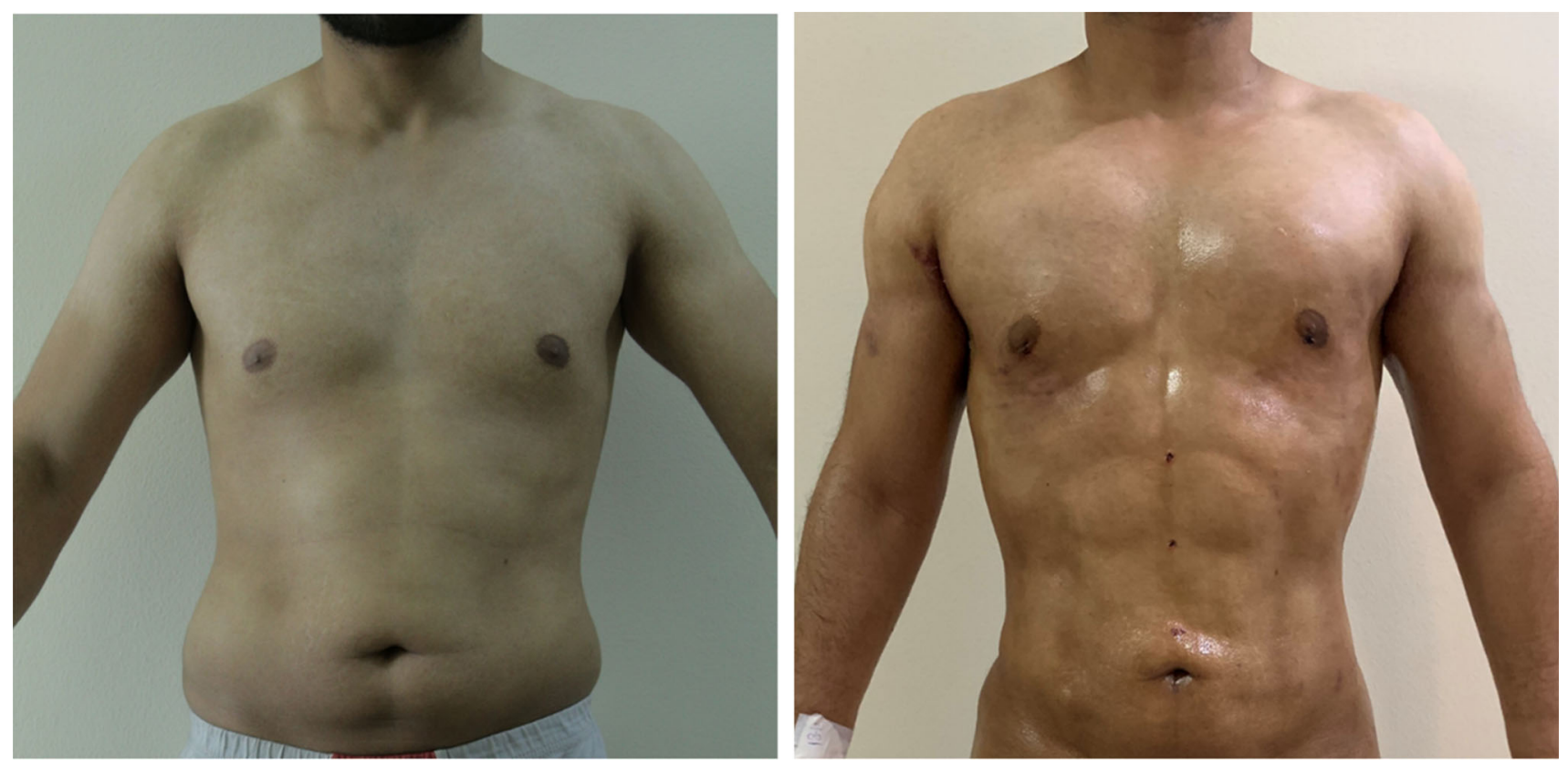

Fig. 4 Male patient, 25 years old, lost $20 \mathrm{lbs}$, BMI 27, underwent 360 liposculpture with fat grafting to the deltoid and pectoralis muscle. The results are ten days post-surgery
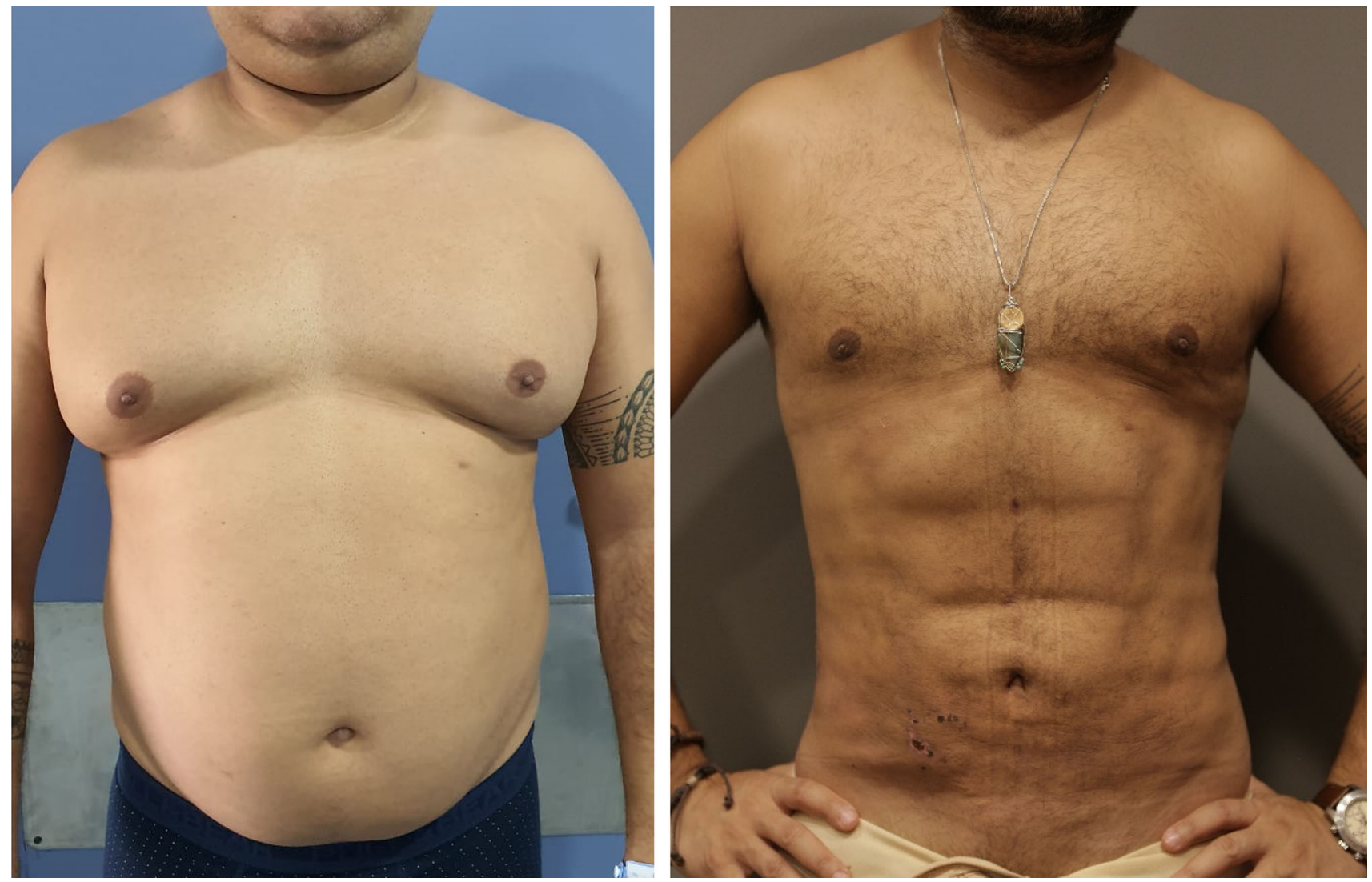

Fig. 5 Mildly obese patient with a BMI $34 \mathrm{~kg} / \mathrm{m}^{2}$ showing excellent skin retraction and redraping. Results are just two months post-surgery. Fat grafting pectoral region shows much enhancement for the upper torso with a smooth narrowing into the waist, hence; the V-tapered look 
its final destination (Fig. 4). Additionally, liposuction may improve health [13] (Fig. 5).

Funding This research did not receive any specific grant from any funding agency in the public, commercial or not-for-profit sector. All authors have made substantial contributions to all of the following: (1) the conception and design of the study, or acquisition of data, or analysis and interpretation of data; (2) drafting the article or revising it critically for important intellectual content; (3) final approval of the version to be submitted. The manuscript, including related data, figures, and tables, has not been previously published and is not under consideration elsewhere.

\section{Compliance with Ethical Standards}

Conflict of interest All authors have no things to disclose.

Human and Animal Right Statements This article does not contain any studies with human participants or animals performed by any of the authors.

Informed Consent A verbal and written consent was obtained from all patients.

\section{References}

1. Lockwood T (1991) Transverse flank-thigh buttock lift with superficial fascial suspension. Plast Reconst Surg 87(6):1019-1027

2. Carloni R, De Runz A, Chaput B, Herlin C, Girardi P, Watieri E, Bertheuril N (2016) Circumferential contouring of the lower trunk: indications, operative techniques, and outcomes-a systematic review. Aesthet Plast Surg 40:652-668

3. Hoyos AE, Millard JA (2007) VASER-assisted high-definition liposculpture. Aesthet Surg J 27(6):594-604
4. Strong AL, Cederna PS, Rubin JP, Coleman SR, Levi B (2015) The current state of fat grafting: a review of harvesting, processing, and injection techniques. Plast Reconstr Surg 136(4):897-912

5. Rieck B, Schlaak S (2003) In vivo tracking of rat preadipocytes after autologous transplantation. Ann Plast Surg 51:294-300

6. Nagy MW, Vanek PF Jr (2014) A multicenter, prospective, randomized, single-blind, controlled clinical trial comparing VASER-assisted lipoplasty and suction-assisted lipoplasty. Plast Reconstr Surg 129(4):681e-689e

7. Sasaki GH, Lopez Ulloa AT (2011) Power-assisted liposuction (PAL) vs. traditional liposuction: quantification and comparison of tissue shrinkage and tightening. In: Advanced techniques in liposuction and fat transfer, Nikolay Serdev. IntechOpen. https:// doi.org/10.5772/24288

8. Abboud MH, Abboud NM, Dibo SA (2016) Brachioplasty by power-assisted liposuction and fat transfer: a novel approach that obviates skin excision. Aesthet Surg J 36(8):908-917

9. Rahrovan S, Fanian F, Mehryan P, Humbert P, Firooz A (2018) Male versus female skin: what dermatologists and cosmeticians should know. Int J Womens Dermatol 4(3):122-130

10. Marshall SA, Senadheera SN, Parry LJ, Girling JE (2017) The role of relaxin in normal and abnormal uterine function during the menstrual cycle and early pregnancy. Reprod Sci 24(3):342-354

11. Turan V, Colluoglu C, Turkyilmaz E, Korucuoglu U (2011) Prevalence of diastasis recti abdominis in the population of young multiparous adults in Turkey. Ginekol Pol 82(11):817-821

12. Greco RJ (1997) Massive liposuction in the moderately obese patients: a preliminary study. Aesthet Surg J 17(2):87-90

13. Benatti FB, Lira FS, Oyama LM, do Nascimento CM, Lancha AH Jr (2011) Strategies for reducing body fat mass: effects of liposuction and exercise on cardiovascular risk factors and adiposity. Diabetes Metab Syndr Obes 4:141-154

Publisher's Note Springer Nature remains neutral with regard to jurisdictional claims in published maps and institutional affiliations. 